VOL. $3(1970), 277-285$.

\title{
On probabilities of large deviations
}

\section{Vijay K. Rohatgi}

Let $\left\{X_{n}\right\}$ be a sequence of independent identically distributed random variables and let $S_{n}=\sum_{k=1}^{n} X_{k}$. The rate of convergence of probabilities $P\left\{\left|S_{n}\right|>(n \log n)^{1 / r}\right\}$, where $2>r>1$, is studied.

\section{Introduction}

Let $\left\{X_{n}: n \geq 1\right\}$ denote a sequence of independent identically distributed random variables with common distribution function $F$. Write $S_{n}=\sum_{k=1}^{n} x_{k}$. If $F$ belongs to the domain of normal attraction of a stable law $V(x)$ with characteristic exponent $\alpha(1<\alpha<2)$ then for some $a>0$ and some $A_{n}$

$$
\lim _{n \rightarrow \infty} P\left\{a^{-1} n^{-1 / \alpha_{S}}{ }_{n}-A_{n} \leq x\right\}=V(x)
$$

(See, for example [3, p. 181].) If, moreover, $E X_{1}=0$ then the constants $A_{n}$ may be taken to be zero and it follows that $a^{-1} n^{-1 / \alpha}(\log n)^{-1 / \alpha_{S}} S_{n} 0$ in probability. Clearly

$$
P\left\{\left|S_{n}\right|>\varepsilon(n \log n)^{1 / \alpha}\right\} \geq P\left\{\left|S_{n}\right|>\varepsilon(n \log n)^{1 / r}\right\}
$$

for $0<r<\alpha$ and it follows that $(n \log n)^{-1 / r_{S}} S_{n} 0$ in probability.

Received 4 July 1970. Research supported by the National Science Foundation, Contract No. NSF-GP-9396. 
The probability $P\left\{\left|S_{n}\right|>\varepsilon(n \log n)^{1 / r}\right\}$ or either of its one sided components is called a probability of large deviation [7]. If $X$ has a finite variance the probability $P\left\{\left|S_{n}\right|>\varepsilon(n \log n)^{1 / 2}\right\}$ is called a probability of moderate deviation (see [1], [5], [6]). We remark that we do not assume that $E X_{2}^{1}<\infty$ but only that $E\left|X_{1}\right|^{r}<\infty$ for $l<r<2$.

In what follows we will assume that $\left\{x_{n}\right\}$ is a sequence of independent identically distributed random variables with common distribution function $F$. A median for the random variable $X$ is denoted by $\operatorname{med}(X)$ and $\lg x$ is the function defined by $\lg _{g} x=\log _{e} x$ for $x>1,=0$ otherwise. $c$ denotes a generic (positive) constant and $1<r<2$.

\section{Results}

Lemmas 1 and 2 are stated here for completness. For proofs we refer to $[1]$.

LEMMA 1. For $r \geq 1, E|X|^{r}<\infty$ if and only $\sum n^{r-1}(\log n)^{r} P\{|X|>n \log n\}<\infty$.

LEMMA 2. Let $\left\{A_{n}\right\}$ be a sequence of independent events. If $\sum P A_{n}<\infty$ then

$$
P\left\{U_{n} A_{n}\right\} \geq \sum_{n} P A_{n}-\sum_{n} P A_{n} \sum_{j=n+1}^{\infty} P A_{j} .
$$

THEOREM. For $1<r<2$ the following statements are equivalent:

(a) $E X_{1}=0, E\left|X_{1}\right|^{r}<\infty$;

(b) $\sum n^{-1} \log n P\left\{\left|S_{n}\right|>\varepsilon(n \log n)^{1 / x}\right\}<\infty$ for all $\varepsilon>0$;

(c) $\sum n^{-1} \log n P\left\{\max _{1 \leq k \leq n}\left|S_{k}\right|>\varepsilon(n \lg n)^{1 / r}\right\}<\infty$ for all $\varepsilon>0$; 
(d) $\sum n^{-1} P\left\{\sup _{k \geq n}\left|S_{k} /\left(k l_{g} k\right)^{1 / r}\right|>\varepsilon\right\}<\infty$ for all $\varepsilon>0$.

Proof. The methods of proof parallel those used in [1] and are fairly standard.

\section{Equivalence of $(a)$ and $(b)$}

It is convenient to make the proofs for symmetrized random variables $x_{n}^{s}, n=1,2, \ldots$ and then use the weak symmetrization inequalities $[4$, p. 245] to transfer to the required results.

Suppose that (a) holds and write $S_{n}^{s}=\sum_{1}^{n} x_{k}^{s}$. Note that $E\left|X_{1}^{8}\right|^{r}<\infty . \quad$ (See [4, p. 246].) Define

$$
X_{k n}^{s}=\left\{\begin{array}{lll}
X_{k}^{s} & \text { if }\left|X_{k}^{s}\right|<\varepsilon(n \lg n)^{1 / r} & \\
0 & \text { otherwise, } & 1 \leq k \leq n
\end{array}\right.
$$

and let $s_{n n}^{s}=\sum_{l}^{n} X_{k n}^{s}$. Since

$$
P\left\{\left|s_{n}^{s}\right|>\varepsilon\left(n \lg _{g}\right)^{1 / r}\right\} \leq n P\left\{\left|X_{1}^{s}\right|>\varepsilon\left(n \lg _{g}\right)^{1 / r}\right\}+P\left\{\left|S_{n n}^{s}\right|>\varepsilon\left(n \lg _{g}\right)^{1 / r}\right\},
$$

and from Lemma 1, $E\left|X_{1}^{s}\right|^{p}<\infty$ implies $\operatorname{LlgnP}\left\{\left|X_{1}^{s}\right|>\varepsilon(n \lg n)^{1 / r}\right\}<\infty$, it just remains to show that $\sum \frac{\lg n}{n} P\left\{\left|S_{n n}^{s}\right|>\varepsilon(n \lg n)^{1 / r}\right\}<\infty$.

From Markov's inequality $[4, p .148]$ we have 


$$
\begin{aligned}
& \sum \frac{\lg n}{n} P\left\{\left|S_{n n}^{s}\right|>\varepsilon(n I g n)^{1 / r}\right\}
\end{aligned}
$$

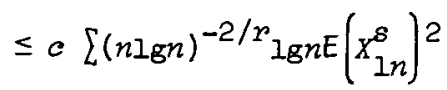

$$
\begin{aligned}
& \leq c \sum_{n} n^{-2 / r}(\lg n)^{1-2 / r} \sum_{k}(k \lg k)^{2 / r} P\left\{(k-1) l_{B}(k-1) \leq\left|X^{s}\right|^{r}<k \lg _{k}\right\} \\
& =c \sum_{k}(k \lg k)^{2 / r_{P}}\left\{(k-1) \lg (k-1) \leq\left|X^{s}\right|^{r}<k \lg k\right\} \sum_{n=k}^{\infty} n^{-2 / r}(\lg n)^{1-2 / r} \\
& \leq c \sum_{k}(k \lg k) P\left\{(k-1) \lg (k-1) \leq\left|x^{s}\right|^{r}<k \lg k\right\} \\
& <\infty \text {. }
\end{aligned}
$$

The last series converges because $E\left|X_{1}^{s}\right|^{r}<\infty$.

$$
\begin{aligned}
& \text { Now note that } E\left|X_{1}\right|^{r}<\infty, \\
& E X_{1}=0 \Rightarrow n^{-1 / r_{S}} S_{n} \stackrel{P}{\longrightarrow} 0 \Rightarrow(n \lg n)^{-1 / r_{S}} S_{n} \stackrel{P}{\longrightarrow} 0 \Rightarrow \operatorname{med}\left(\frac{S_{n}}{(n \log n)^{1 / r}}\right) \rightarrow 0 .
\end{aligned}
$$

It is now easy to complete the proof of $(a) \Rightarrow(b)$ by a simple use of weak symetrization inequalities.

Next suppose that (b) holds. By the symmetrization inequalities $\sum n^{-1} \operatorname{lgn}\left\{S_{n}^{s}>\varepsilon(n \lg n)^{1 / r}\right\}<\infty$ where $S_{n}^{s}$, as before, is the sum of the symmetrized random variables. We first show that $(n \lg n)^{-1 / r_{S} s} \stackrel{P}{\longrightarrow} 0$. If not, there exists an $\varepsilon_{0}>0$ such that either $P\left\{S_{n_{k}}^{s}>\varepsilon_{0}\left(n_{k} \lg n_{k}\right)^{1 / r}\right\}>\varepsilon_{0}$ or $P\left\{S_{n_{k}}^{s}<-\varepsilon_{0}\left(n_{k} \lg n_{k}\right)^{1 / r}\right\}>\varepsilon_{0}$ for infinitely many $k$. For the sake of argument assume $P\left\{S_{n_{k}}^{s}>\varepsilon_{0}\left(n_{k} \lg n_{k}\right)^{1 / r}\right\}>\varepsilon_{0}$ for infinitely many $k$ and choose $n_{k+1}>2 n_{k}$. Then for each $j, n_{k} \leq j<2 n_{k}$, we have

$$
(j \perp g j)^{1 / r}<\left(2 n_{k} \lg \left(2 n_{k}\right)\right)^{1 / r} \leq 2^{2 / r}\left(n_{k} \lg n_{k}\right)^{1 / r}
$$

and 


$$
\begin{aligned}
P\left\{S_{j}^{s}>\varepsilon_{0} \frac{(j l g j)^{I / r}}{2^{2 / r}}\right\} & \geq P\left\{S_{j}^{s}>\varepsilon_{0}\left(n_{k} \lg n_{k}\right)^{1 / x}\right\} \\
& \geq \frac{1}{2} P\left\{S_{n_{k}}^{s}>\varepsilon_{0}\left(n_{k} l g n_{k}\right)^{1 / r}\right\} \\
& \geq \frac{\varepsilon_{0}}{2} .
\end{aligned}
$$

It follows that

$$
\begin{aligned}
\sum \frac{\lg n}{n} P\left\{S_{n}^{s}>\frac{\varepsilon_{0}}{2^{2 / r}}(n \lg n)^{1 / r}\right\} & \geq \sum_{k} \sum_{i=n_{k}}^{2 n_{k}} \frac{\lg i}{i} P\left\{S_{i}^{s}>\frac{\varepsilon_{0}}{2^{2 / r}}(i \lg i)^{1 / r}\right\} \\
& \geq \frac{\varepsilon_{0}}{2} \sum_{k} \sum_{i=n_{k}}^{2 n_{k}} \frac{\lg i}{i}=\infty .
\end{aligned}
$$

This contradiction shows that $(n \lg n)^{-1 / r_{S} s} S_{n} \rightarrow 0$ in probability. By the degenerate convergence criterion [4, p. 317] we get $n P\left\{X_{n}^{s}>\varepsilon(n \lg n)^{1 / r}\right\} \rightarrow 0$. Following Erdös [2] we write $A_{k}=\left\{X_{k}^{s}>\varepsilon(n \lg n)^{1 / r}\right\}$ and $B_{k}=\left\{\sum_{j \neq k}^{n} X_{j}^{s} \geq 0\right\}$ and see that

$$
\begin{aligned}
P\left\{S_{n}^{s}>\varepsilon(n \lg n)^{1 / r}\right\} & \geq P\left\{\bigcup_{k=1}^{n}\left(A_{k} \cap B_{k}\right\}\right\} \\
& \geq \sum \sum_{i}^{n}\left[P B_{i}-n P A_{i}\right] \\
& \geq n P A_{i}\left|\frac{1}{2}-n P A_{i}\right| .
\end{aligned}
$$

Thus for $\delta>0$ and large $n$ we have

$$
P\left\{S_{n}^{s}>\varepsilon(n \lg n)^{1 / 2}\right\} \geq\left(\frac{1}{2}-\delta\right) n P A_{i} .
$$

It follows that

$$
\infty>\sum n^{-1} \operatorname{lgn}\left\{S_{n}^{s}>\varepsilon(n \lg n)^{1 / r}\right\} \geq c \sum \operatorname{lgn} P\left\{X_{1}^{s}>\varepsilon(n \lg n)^{1 / x}\right\} .
$$

By Lemma 1, we obtain $E\left|X_{1}^{s}\right|^{p}<\infty$ and thus $E\left|X_{1}\right|^{r}<\infty$ by Corollary 2 [4, 
p. 246].

To show that $E X_{1}=0$ we only have to note that

$$
E\left|X_{1}\right|^{r}<\infty=n^{-1} S_{n} \stackrel{a . s .}{\longrightarrow} E X_{1} \text { and }(n \lg n)^{-1 / r_{S}} \stackrel{P}{\longrightarrow} 0 \text {. }
$$

Equivalence of (b) and (c)

The $(c) \Rightarrow(b)$ part is trivial and the $(b) \Rightarrow$ (c) part follows from Lévy's inequality $[4, p .247]$ and the fact that $(b) \Rightarrow(n \lg n)^{-1 / x_{S}}{ }_{n} \stackrel{P}{\rightarrow} 0$.

Equivalence of (d) and (a)

We first show that $(a)$ and $(b) \Rightarrow(d)$. Choose $i$ such that $2^{i} \leq n<2^{i+1}$ and again consider the symmetrized random variables $x_{k}^{s}$ and $S_{n}^{s}$. We have

$$
\begin{aligned}
P\left\{S_{n}^{s}>\varepsilon(n \lg n)^{1 / r}\right\} & \geq P\left\{S_{n}^{s}>\varepsilon\left(2^{i+1} l_{g} 2^{i+1}\right)^{1 / r}\right\} \\
& \geq \frac{1}{2} P\left\{S_{2^{i}}^{s}>\varepsilon\left(2^{i+1}{ }_{1 g} 2^{i+1}\right)^{1 / r}\right\} \\
& \geq \frac{1}{2} P\left\{S_{2^{s}}^{s}>\varepsilon 2^{2 / r}\left(2^{i} \lg ^{i}\right)^{1 / r}\right\} .
\end{aligned}
$$

Using once again the symmetrization inequalities, we have

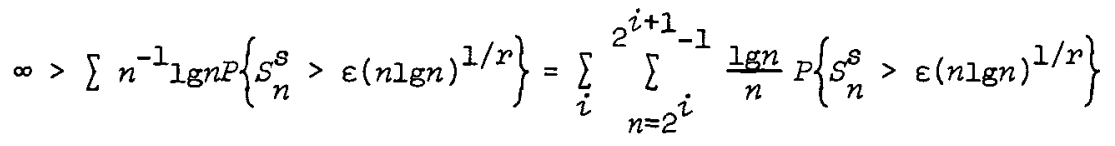

$$
\begin{aligned}
& \geq \frac{1}{4} \sum_{i} \lg 2^{i} P\left\{S_{2^{i}}>2^{2 / r} \varepsilon\left(2^{i} \lg ^{i}\right)^{1 / r}\right\} \text {, }
\end{aligned}
$$

so that 


$$
\begin{aligned}
& \sum_{n} n^{-1} P\left\{\sup _{k \geq n} \frac{s_{k}^{s}}{\left(k I_{g} k\right)^{1 / r}}>\varepsilon\right\} \\
& \leq c \sum_{i} P\left\{\sup _{k \geq 2} \frac{S_{k}^{s}}{(k l g k)^{1 / r}}>\varepsilon\right\} \\
& \leq c \sum_{i} \sum_{j=i}^{\infty} P\left\{\max _{2^{i} \geq k<2^{j+1}} \frac{s_{k}^{\varepsilon}}{(k l g k)^{1 / x}}>\varepsilon\right\} \\
& \leq c \sum_{i} \sum_{j=i}^{\infty} P\left\{S_{2^{j+1}}^{s}>\varepsilon\left(2^{j} l_{g} 2^{j}\right)^{l / r}\right\} \quad \text { (Lévy's inequality) } \\
& =c \sum_{j=1}^{\infty} j P\left\{S_{2^{j+1}}>\varepsilon\left(2^{j} l g 2^{j}\right)^{1 / x}\right\} \\
& \leq c \sum_{j} \lg _{g} 2^{j+1}\left\{S_{2^{j+1}}>\varepsilon 2^{-2 / r}\left(2^{j+1}{ }_{1 g 2^{j+1}}\right)^{1 / r}\right\} \\
& \leq c \sum_{n} n^{-1} \lg n\left\{\left\{S_{n}^{s}>\varepsilon \cdot 2^{-4 / r}(n \lg n)^{1 / r}\right\}\right. \\
& <\infty \text {. }
\end{aligned}
$$

It just remains to use the weak symetrization inequalities and the fact that $(n \lg n)^{-1 / r_{S}} S_{n} \stackrel{P}{\longrightarrow} 0$ to see that $(a)$ and $(b) \Rightarrow(d)$.

Next we show that $(d) \Rightarrow(a)$.

Let $A_{k}=\left\{\left|X_{k}\right|>\varepsilon\left(k I_{g} k\right)^{l / r}\right\}$. Then the events $A_{k}$ are independent and satisfy

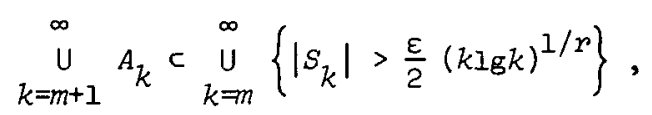

so that

$$
\begin{aligned}
P\{\underbrace{\infty}_{m+1} A_{k}\} & \leq P\left\{\underset{k=m}{U}\left\{\left|S_{k}\right|>\frac{\varepsilon}{2}(k 1 g k)^{1 / r}\right\}\right\} \\
& =P\left\{\sup _{k \geq m}\left|\frac{S_{k}}{(k l g k)^{1 / r}}\right|>\frac{\varepsilon}{2}\right\}
\end{aligned}
$$


which $\rightarrow 0$ as $m \rightarrow \infty$ because of the fact that the sequence $P\left\{\sup _{k \geq m}\left|\frac{S_{k}}{(k l g k)^{1 / x}}\right|>\frac{\varepsilon}{2}\right\}$ is non-increasing in $m$ and (d) holds. An application of the Borel zero-one criterion $[4, p .228]$ now shows that

$$
\sum_{k=1}^{\infty} P\left\{\left|X_{1}\right|>\varepsilon(k \lg k)^{1 / r}\right\}=\sum_{k=1}^{\infty} P A_{k}<\infty \text {. }
$$

By Lemma 2 therefore

$$
\begin{aligned}
& \infty>\sum_{n=2}^{\infty} n^{-1}\left\{\left\{\sup _{k \geq n}\left|\frac{s_{k}}{(k \lg k)^{I / r}}\right|>\frac{\varepsilon}{2}\right\}\right. \\
& \geq \sum_{n=2}^{\infty} n^{-1}\left\{\left\{\bigcup_{k=0}^{\infty}\left\{\left|x_{n+k}\right|>\varepsilon((n+k) \lg (n+k))^{1 / r}\right\}\right\}\right. \\
& \geq c \sum^{-1} \sum_{k=0}^{\infty} P\left\{\left|x_{n+k}\right|>\varepsilon((n+k) \lg (n+k))^{1 / r}\right\}(1-n) \\
& \geq c \sum_{k=2}^{\infty} P\left\{\left|x_{1}\right|>\varepsilon(k \lg k)^{1 / r}\right\} \sum_{n=2}^{k} \frac{1}{n} \\
& \geq c \sum_{k} \operatorname{lgk}\left\{\left|x_{1}\right|>\varepsilon(k \lg k)^{1 / r}\right\} .
\end{aligned}
$$

It follows by Lemma 1 that $E\left|X_{1}\right|^{r}<\infty$. From (d) we see that $n^{-1} S_{n} \stackrel{a \cdot s}{\longrightarrow} 0$ so that we must have $E X_{1}=0$. Thus $(d) \Rightarrow(a)$.

This completes the proof of the theorem.

\section{References}

[1] James Avery Davis, "Convergence rates for probabilities of moderate deviations", Ann. Math. Statist. 39 (1968), 2016-2028.

[2] P. Erdös, "On a theorem of Hsu and Robbins", Ann. Math. Statist. 20 (1949), 286-291. 
[3] B.V. Gnedenko and A.N. Kolmogorov, Limit distributions for sums of independent random variables (Translated and annotated by K.L. Chung with an appendix by J.L. Doob. Addison-Wesley, Cambridge, Massachussetts; 1954).

[4] Michel Loève, Probability theory, 3rd ed. (Van Nostrand, Princeton, New Jersey; Toronto, Ontario; London; 1963).

[5] V.K. Rohatgi, "On the rate of convergence of probabilities of moderate deviations", J. Austral. Math. Soc. 11 (1970), 91-94.

[6] Herman Rubin and J. Sethuraman, "Probabilities of moderate deviations", Sankhyā Ser. A 27 (1965), 325-346.

[1] J. Sethuraman, Probabizities of deviations (Indian Statistical Institute Report no. 5, 1966).

The Catholic University of America, Washington, DC, USA. 